\title{
INDECOMPOSABLE SUMMANDS OF FOULKES MODULES
}

\author{
EUGENIO GIANNELLI AND MARK WILDON
}

\begin{abstract}
In this paper we study the modular structure of the permutation module $H^{\left(2^{n}\right)}$ of the symmetric group $S_{2 n}$ acting on set partitions of a set of size $2 n$ into $n$ sets each of size 2 , defined over a field of odd characteristic $p$. In particular we characterize the vertices of the indecomposable summands of $H^{\left(2^{n}\right)}$ and fully describe all of its indecomposable summands that lie in blocks of $p$-weight at most two. When $2 n<3 p$ we show that there is a unique summand of $H^{\left(2^{n}\right)}$ in the principal block of $S_{2 n}$ and that this summand exhibits many of the extensions between simple modules in its block.
\end{abstract}

\section{INTRODUCTION}

The symmetric group $S_{2 n}$ acts on the collection of all set partitions of $\{1, \ldots, 2 n\}$ into $n$ sets each of size two. Let $H^{\left(2^{n}\right)}$ denote the corresponding permutation representation of $S_{2 n}$, defined over a field $F$ of odd prime characteristic $p$. (Equivalently, $H^{\left(2^{n}\right)}$ is the $F S_{2 n}$-module induced from the trivial representation of the imprimitive wreath product $S_{2} \imath S_{n} \leq S_{2 n}$.) We call $H^{\left(2^{n}\right)}$ a Foulkes module. In the main theorem of [7] the authors used results on the indecomposable summands of Foulkes modules to determine certain decomposition numbers of the symmetric group. In this paper we study the structure of Foulkes modules more closely. In particular, we characterize the vertices of the indecomposable summands of each $H^{\left(2^{n}\right)}$ and give a precise description of all summands in blocks of $p$-weight at most two.

Let $Q_{t}$ be a Sylow $p$-subgroup of $S_{2} \prec S_{t p}$ and let $Q_{0}$ be the trivial group. By [7, Theorem 1.2], if $U$ is an indecomposable summand of $H^{\left(2^{n}\right)}$ then $U$ has vertex $Q_{t}$ for some $t \in \mathbf{N}_{0}$. Our first main theorem gives the converse.

Theorem 1.1. Let $n \in \mathbf{N}$. For all $t \in \mathbf{N}_{0}$ such that $t \leq n / p$ there is an indecomposable summand of $H^{\left(2^{n}\right)}$ with vertex $Q_{t}$.

To state the second main theorem we need some more definitions from [7]. When defined over a field of characteristic zero, $H^{\left(2^{n}\right)}$ has ordinary character $\sum \chi^{2 \lambda}$ where the sum is over all partitions $\lambda$ of $n$, and $2 \lambda$ is the partition

2010 Mathematics Subject Classification. Primary 20C30. Secondary 05A17, 20C20.

The first author is supported by the London Mathematical Society Postdoctoral Mobility Grant PMG14-15 02. 
obtained from $\lambda$ by doubling each part. (For an elegant proof of this fact with minimal prerequisites, see [11.) We say that such partitions are even. Given a $p$-core $\gamma$, let $w(\gamma)$ be the minimum number of $p$-hooks that, when added to $\gamma$, give an even partition. Let $\mathcal{E}(\gamma)$ be the set of even partitions that can be obtained by adding $w(\gamma) p$-hooks to $\gamma$. For example, if $p=3$, then $w((3,1))=2$ and $\mathcal{E}((3,1))=\{(6,4),(6,2,2),(4,4,2)\}$. Let $B(\gamma, w)$ denote the $p$-block of $S_{n}$ with $p$-core $\gamma$ and $p$-weight $w$, where $n=|\gamma|+w p$. As a convenient shorthand, we write $\nu \in B(\gamma, w)$ to mean that the partition $\nu$ has $p$-core $\gamma$ and $p$-weight $w$. Let $S^{\mu}$ denote the Specht module labelled by the partition $\mu$. For $\mu$ a $p$-regular partition, let $P^{\mu}$ denote the projective cover of the simple $F S_{n}$-module $D^{\mu}$, defined in [14, Corollary 12.2] as the unique top composition factor of $S^{\mu}$. Finally let $d_{\mu \nu}=\left[S^{\mu}: D^{\nu}\right]$.

Theorem 1.2. Let $n \in \mathbf{N}$. Let $\gamma$ be a p-core.

(i) There is a summand of $H^{\left(2^{n}\right)}$ in $B(\gamma, 0)$ if and only if $\gamma$ is even. In this case the unique summand is the simple projective Specht module $S^{\gamma}$.

(ii) There is a summand of $H^{\left(2^{n}\right)}$ in $B(\gamma, 1)$ if and only if $w(\gamma)=1$. In this case $\mathcal{E}(\gamma)=\left\{2 \lambda, 2 \lambda^{\prime}\right\}$ for partitions $\lambda, \lambda^{\prime}$ with $\lambda \triangleleft \lambda^{\prime}$, and the unique summand is $P^{2 \lambda^{\prime}}$.

(iii) There is a summand of $H^{\left(2^{n}\right)}$ in $B(\gamma, 2)$ if and only if $w(\gamma)=0$ or $w(\gamma)=2$. If $w(\gamma)=2$ the unique summand is $P^{2 \lambda}$, where $2 \lambda$ is the unique maximal element of $\mathcal{E}(\gamma)$. If $w(\gamma)=0$ the unique summand has vertex $Q_{1}$ and its Green correspondent is $P \otimes S^{\gamma}$ as a representation of $N_{S_{2 n}}\left(Q_{1}\right) / Q_{1} \cong N_{S_{2 p}}\left(Q_{1}\right) / Q_{1} \times S_{n-2 p}$, where $P$ is the projective cover of the trivial $F N_{S_{2 p}}\left(Q_{1}\right) / Q_{1}$-module.

If $t=\lfloor n / p\rfloor$ then $Q_{t}$ is a Sylow $p$-subgroup of $S_{2} 2 S_{n}$. The projective cover of the trivial representation of $N_{S_{2 n}}\left(Q_{t}\right) / Q_{t}$ has vertex $Q_{t}$ as a representation of $N_{S_{2 n}}\left(Q_{t}\right)$; its Green correspondent is a summand of $H^{\left(2^{n}\right)}$ with vertex $Q_{t}$ lying in the principal block of $S_{2 n}$. This summand is an example of a Scott module: see [8] or [3] for their definition and basic properties. Our third main theorem describes these summands when $2 n<3 p$.

Theorem 1.3. Let $2 k<p$ and let $2 n=2 p+2 k$. There is a unique summand $U$ of $H^{\left(2^{n}\right)}$ in the principal block of $S_{2 n}$. This summand is the $S$ cott module with vertex $Q_{1}$. The module $U$ has three Loewy layers and

$$
\operatorname{soc} U \cong \operatorname{top} U \cong \bigoplus_{\substack{2 \nu \in B((2 k), 2) \\ 2 \nu \neq\left(2 k, 2^{p}\right)}} D^{2 \nu} .
$$

The Loewy layers of $U$ are shown in Figure 1 overleaf, where each edge shows an element of $\mathrm{Ext}^{1}$ exhibited by this module.

Our final theorem counts the summands of $H^{\left(2^{n}\right)}$ which, like the Scott module summand, have the largest possible vertex. 


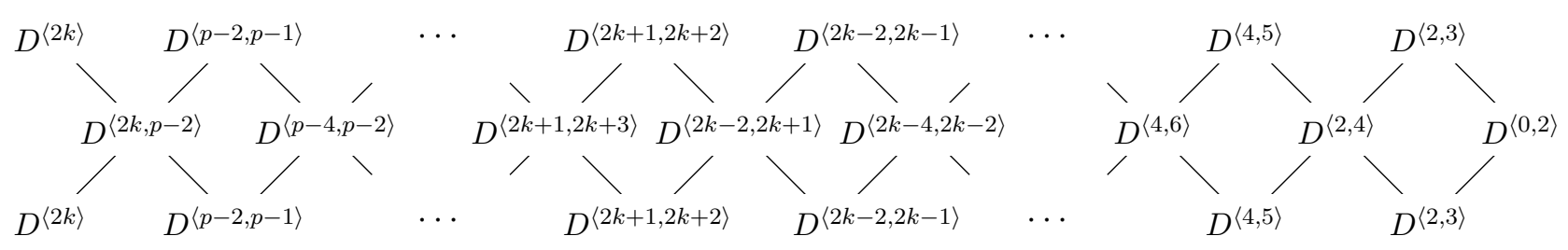

FiguRE 1. The three Loewy layers of the unique principal block summand of $H^{\left(2^{n}\right)}$ when $n=2(p+k)$ and $2<2 k<p-1$. The labels of simple modules are defined in Section 7. If $2 k \in\{0,2, p-1\}$ then the structure is the same but minor changes must be made to the labels: see Figure 5 in Section 7 .

Theorem 1.4. Let $t=\lfloor n / p\rfloor$ and let $r=n-t p$. The number of indecomposable summands of $H^{\left(2^{n}\right)}$ with vertex $Q_{t}$ is equal to the number of $p$-core partitions that can be obtained by removing p-hooks from even partitions of $2 r$. Each such summand lies in a different block of $S_{2 n}$.

In particular we note that if $0 \leq 2 r<p$ then the number of indecomposable summands of $H^{\left(2^{n}\right)}$ with vertex $Q_{t}$ is simply the number of even partitions of $2 r$. Another easy corollary of our theorems is the following.

Corollary 1.5. The unique non-trivial indecomposable Foulkes module is $H^{\left(2^{2}\right)}$ when $p=3$.

Proof. By Theorem 1.1 there are summands of $H^{\left(2^{n}\right)}$ with vertices $Q_{0}$ and $Q_{1}$ whenever $n \geq p$. When $4 \leq n<p$ the partitions $(2 n),(2 n-2,2)$ and $(2 n-4,4)$ are not all in the same block, so $H^{\left(2^{n}\right)}$ has summands in two different blocks. By Theorem 1.2 if $p=3$ then $H^{\left(2^{2}\right)}$ is indecomposable; if $p>3$ then $H^{\left(2^{2}\right)}=S^{(4)} \oplus S^{(2,2)}$. If $p=3$ then $H^{\left(2^{3}\right)}$ has $S^{(4,2)}$ as the unique summand in its block; if $p=5$ then $H^{\left(2^{3}\right)}$ has $S^{(2,2,2)}$ as the unique summand in its block; if $p>5$ then $H^{\left(2^{3}\right)}=S^{(6)} \oplus S^{(4,2)} \oplus S^{(2,2,2)}$.

The results in this paper show that the behaviour of Foulkes modules is very different from the better studied Young permutation modules (see, for instance, [5], 44, [10] and [12]). In particular, by Theorem 1.2, each Foulkes module has at most one summand in each block of weight at most two. The Young permutation modules $M^{(r-s, s)}$ also have multiplicity-free ordinary characters, but it follows easily from [10, Theorem 3.3] that if $p$ is odd, $0 \leq c<p$ and $p \leq j \leq p+c / 2$ then $M^{(2 p+c-j, j)}$ always has two summands, namely the Young modules $Y^{(2 p+c)}$ and $Y^{(p+c, p)}$, in the principal block of $S_{2 p+c}$. By Corollary 1.5 there is only one non-trivial indecomposable Foulkes module, whereas $M^{(p-1,1)}$ is always indecomposable. (The indecomposable Young permutation modules are classified in [4, Theorem 2].) It would be 
interesting to know the decomposition of further permutation modules for symmetric groups in prime characteristic.

Outline. In $\S 2$ we give the prerequisite material from [7]. In $\S 3$ we find $w(\gamma)$ for a special class of $p$-cores $\gamma$. This result is used in the proof of Theorem 1.1. In $\S 4$ we recall Richards' results [17] on decomposition numbers in blocks of $p$-weight 2 , and show that even partitions of $p$-weight 2 have a surprisingly simple characterization using his definitions. These results are used in the proof of Theorem 1.2(iii) and Theorem 1.3 . In $\S 5, \S 6$ and $\S 7$ we prove Theorem 1.1, Theorem 1.2 and Theorem 1.3 respectively. Theorem 1.4 is obtained as a corollary of Theorem 1.2 in $\S 6$. We end in $\S 8$ with an example to illustrate these theorems. The proofs in $\S 3$ and $\S 4$ are the most technical in this paper; we suggest the reader returns to the proofs after seeing the applications.

\section{Prerequisites}

The following results have short proofs using [7]. For background on local representation theory we refer the reader to [1].

Proposition 2.1. Let $\gamma$ be a p-core partition and let $2 n=|\gamma|+p w$. The Green correspondence induces a bijection between the indecomposable summands of $H^{\left(2^{n}\right)}$ in $B(\gamma, w)$ with vertex $Q_{t}$ and the indecomposable projective summands of $H^{\left(2^{n-t p}\right)}$ in $B(\gamma, w-2 t)$.

Proof. Let $U$ be an indecomposable non-projective summand of $H^{\left(2^{n}\right)}$ in $B(\gamma, w)$. By [7, Theorem 1.2], $U$ has $Q_{t}$ as a vertex for some $t \in \mathbf{N}$ and its Green correspondent has a tensor factorization $V \otimes W$ as a representation of $\left(N_{S_{2 t p}}\left(Q_{t}\right) / Q_{t}\right) \times S_{2(n-t p)}$. Here $V$ and $W$ are projective and $W$ is an indecomposable summand of $H^{\left(2^{n-t p}\right)}$. By [7, Theorem 2.7], which is proved using the results in [3], $W$ lies in the block $B(\gamma, w-2 t)$ of $S_{2(n-t p)}$. The map sending $U$ to $W$ therefore has the required properties.

Proposition 2.2. Let $\gamma$ be a p-core partition and let $2 n=|\gamma|+p w$.

(i) All the summands of $H^{\left(2^{n}\right)}$ in $B(\gamma, w(\gamma))$ are projective.

(ii) If $2 \lambda$ is a maximal element of $\mathcal{E}(\gamma)$ then $\lambda$ is p-regular and $P^{2 \lambda}$ is a summand of $H^{\left(2^{n}\right)}$ lying in $B(\gamma, w(\gamma))$.

(iii) If $P$ is an indecomposable projective summand of $H^{\left(2^{n}\right)}$ in $B(\gamma, w(\gamma))$ then $P=P^{2 \nu}$ for some $2 \nu \in \mathcal{E}(\gamma)$ and the ordinary character of $P$ (defined using Brauer reciprocity) is $\sum_{2 \lambda \in E} \chi^{2 \lambda}$ for some $E \subseteq \mathcal{E}(\gamma)$.

Proof. Part (i) is Proposition 5.1 of [7]. Since this is a key result in this paper, we briefly sketch the proof: if $U$ is a non-projective summand in $B(\gamma, w(\gamma))$ then, by Proposition 2.1 there is a projective summand of $H^{\left(2^{n-t p}\right)}$ 
in $B(\gamma, w(\gamma)-2 t)$ for some $t \in \mathbf{N}$. But this implies that there is an even partition with $p$-core $\gamma$ and $p$-weight $w(\gamma)-2 t$, contradicting the definition of $w(\gamma)$. Part (ii) is Proposition 1.3 of [7]. Part (iii) follows immediately from $(\dagger)$ in the proof of this proposition.

We use the following proposition in the proof of Theorem 1.2 to show that, if $w \leq 2$, then the summand identified in Proposition 2.2(ii) is the unique summand of $H^{\left(2^{n}\right)}$ lying in its block.

Proposition 2.3. Let $\gamma$ be a p-core partition of $n$. If $|\mathcal{E}(\gamma)|<2 w(\gamma)+1$ then there is a unique summand of $H^{\left(2^{n}\right)}$ in $B(\gamma, w(\gamma))$.

Proof. It was proved independently in [17, Theorem 2.8] and [2, Proposition 4.6(i)] that if $\nu$ is a $p$-regular partition of $n$ lying in a block of $p$-weight $w$ then $d_{\mu \nu}>0$ for at least $w+1$ partitions $\mu$. By Proposition 2.2(i), all the summands of $H^{\left(2^{n}\right)}$ in $B(\gamma, w(\gamma))$ are projective. By Brauer reciprocity, the ordinary character of each such summand contains at least $w+1$ distinct irreducible characters. The result now follows from Proposition 2.2(iii).

\section{Even partitions AND the ABACUS}

We make extensive use of James' abacus notation, as defined in [13, page 78]. We number the abacus runners from 0 to $p-1$. By convention, our abaci have beads in all strictly negative positions. We say that beads before the first space are initial. For $r \in \mathbf{N}_{0}$, we define row $r$ to consist of positions $p r, \ldots, p r+p-1$. By a single-step move we mean a move of a bead into a space immediately below it.

It is simple to recognise even partitions on the abacus.

Definition 3.1. Let $A$ be an abacus. We say that beads in positions $\beta$ and $\beta^{\prime}$ of $A$ where $\beta<\beta^{\prime}$ are adjacent beads and form a gap if there are spaces in positions $\beta+1, \ldots, \beta^{\prime}-1$. This gap is odd if $\beta^{\prime}-\beta$ is even and even if $\beta^{\prime}-\beta$ is odd. If $\beta^{\prime}-\beta=1$, then we say that the beads are in adjacent positions.

Lemma 3.2. Let $A$ be an abacus representing a partition $\nu$. Then $\nu$ is even if and only if $A$ has no odd gaps.

The following proposition is helpful in determining $w(\gamma)$. Informally, it states that certain beads on an abacus representing $\gamma$ need not be moved to find a partition in $\mathcal{E}(\gamma)$.

Proposition 3.3. Let $p$ be odd and let $\gamma$ be a p-core represented by an abacus $A$ with first space in position 0 . Let $B$ be an abacus representing a partition $\lambda \in \mathcal{E}(\gamma)$ obtained by a sequence of single-step moves on $A$. If $x \leq-2$ then $B$ has a bead in position $x$. 
Proof. Suppose, for a contradiction, that there exists $x<-1$ such that $B$ has a space in position $x$. Choose $x$ minimal with this property. Thus if $y<x$ then the bead in position $y$ of $A$ remains fixed, and the bead in position $x$ is moved. To avoid an odd gap, the bead in position $x+1$ of $A$ is also moved. By increasing the number of initial beads in $A$ we may assume that $x=p r$ for some $r \in \mathbf{Z}$. Therefore positions $p r$ and $p r+1$ in $B$ have spaces.

Let $b$ be the highest bead on runner 0 of $B$ below position $p r$, and let $c$ be the highest bead on runner 1 of $B$ below position $p r+1$. If $b$ and $c$ are on the same row then we may move $b$ up to position $p r$ and $c$ up to position $p r+1$ without changing the parity of any gap in $B$. (This holds because there is a bead in position $p r-1$, and the gap between this bead and the adjacent bead in $B$ is even.) We therefore obtain an even partition with $p$-core $\gamma$ and strictly smaller $p$-weight, a contradiction.

Assume that $c$ is on a strictly lower row than $b$; the other case is dealt with symmetrically. Let $b^{\prime}$ be the first bead in a greater numbered position than $b$, and let $c^{\prime}$ be the first bead in a greater numbered position than $c$. Suppose that $b^{\prime}$ is on runner $u$ and $c^{\prime}$ is on runner $v$. There are at most $2(p-1)$ spaces between $b$ and $b^{\prime}$ since otherwise $b^{\prime}$ can be moved two rows up without changing the parity of any gaps. Hence either $b^{\prime}$ is on the same row as $b$, in which case $u$ is odd, or $b^{\prime}$ is on the row directly below, in which case $u$ is even. A similar remark applies to $c$ and $c^{\prime}$, swapping odd and even, with one extra case when $v=0$, in which case $c^{\prime}$ is two rows below $c$.

We claim that there is a sequence of bead moves using the beads $b$ and $b^{\prime}$ and beads on runners $u$ and $v$ that gives a new abacus $B^{\star}$, representing a partition of strictly smaller $p$-weight than $\lambda$, such that $B^{\star}$ has no odd gaps. This can be shown by considering the four cases for the parity of $u$ and $v$, splitting the different parity cases into the subcases $u<v$ and $u>v$, as shown in Figure 2, and the equal parity cases into subcases for $u=0$ and $u \neq 0$, as shown in Figure 3. The indicated bead moves deal correctly with the exceptional cases when $u=0, v=0$ or $v=1$ and two runners coincide. (Note that $u=1$ is impossible because then $b^{\prime}=c$ and $b$ and $c$ are in the same row.) If $v=0$ and $u$ is odd then $c^{\prime}$ is two rows below $c$, the bead moves in the top right diagram in Figure 2 apply and a bead is moved into the position vacated by $b$.

We give full details for the case where $B$ is the top-left abacus in Figure 2. Thus $c$ is on a strictly lower row than $b$, and $b^{\prime}$ and $c^{\prime}$ are in the same rows as $b$ and $c$, respectively. Let $s$ be the row of bead $b, t$ be the row of bead $c$ and let $w$ be the $p$-weight of the partition $\lambda$. Let $B^{\prime}$ be the abacus obtained from $B$ by moving beads $b$ and $c$ up to positions $p r$ and $p r+1$ respectively by a 

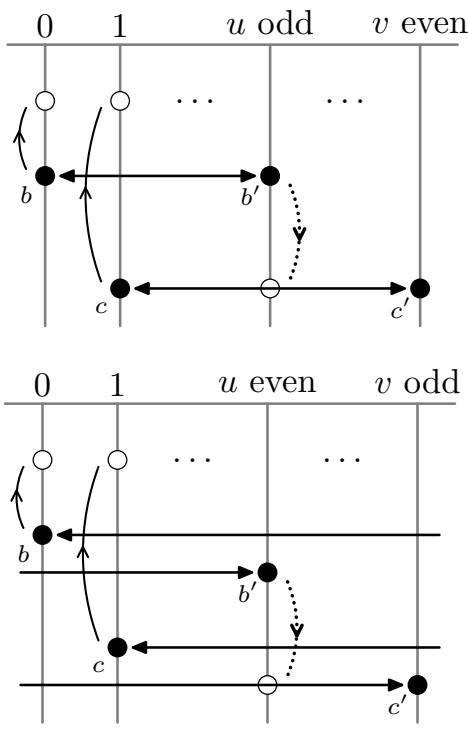
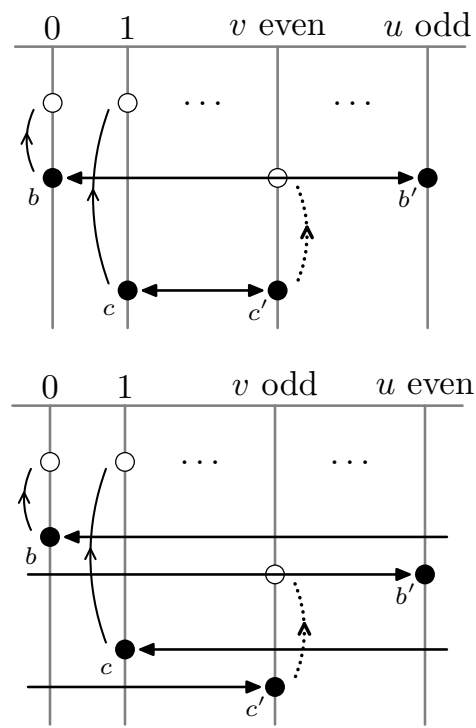

Figure 2. Bead moves on the abacus $B$ used in the proof of Proposition 3.3 when $u$ and $v$ have different parity. White circles show spaces in $B$ that are present by hypothesis and are the target of bead moves; the two adjacent spaces are positions $p r$ and $p r+1$. Thick arrows show even gaps in $B$. Solid curved arrows show moves of the beads $b$ and $c$ on runners 0 and 1, respectively. On runners $u$ and $v$ dotted curved arrows show the overall effect of a sequence of single-step upward or downward moves using the marked bead and the beads between the marked bead and the target space.

sequence of single-step bead moves. Notice that $B^{\prime}$ has no odd gaps in rows $1, \ldots, s-1$, and that $B^{\prime}$ represents a partition of $p$-weight $w-(s-r)-(t-r)$. Let $B^{\prime \prime}$ be the abacus obtained from $B^{\prime}$ by making the unique series of singlestep moves of beads on runner $u$ that has the final effect of swapping bead $b^{\prime}$ with the space in position $p t+u$, in row $t$ on runner $u$. (The bead that ends in position $p t+u$ in $B^{\prime \prime}$ is therefore the lowest bead on runner $u$ of $B^{\prime}$ above this position.) It is easily seen that $B^{\prime \prime}$ has no odd gaps and represents a partition of $p$-weight

$$
w-(s-r)-(t-r)+(t-s)=w-2(s-r) .
$$

This is clearly strictly less than $w$, the $p$-weight of $\lambda$, as required.

Despite its special nature, the following result appears to require the full power of Proposition 3.3 .

Proposition 3.4. Let $\gamma$ be the p-core represented by an abacus having $t$ beads on runner $u$ and $t^{\prime}$ beads on runner $u^{\prime}$, where $u<u^{\prime}$, and no beads on any other runner. 

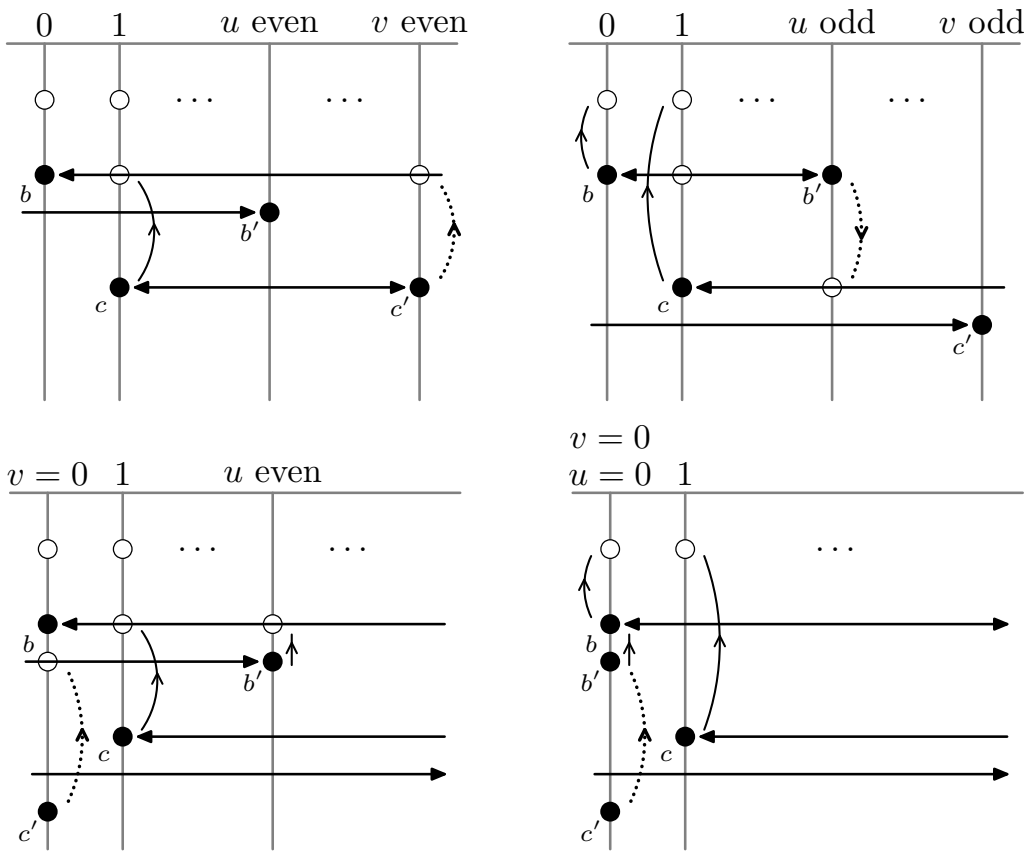

Figure 3. Bead moves on the abacus $B$ used in the proof of Proposition 3.3 when $u$ and $v$ have equal parity. The conventions for arrows are as described in the caption of Figure 2. The cases $u=0, v \neq 0$ and $u=v=0$ are dealt with separately: in the latter case at least four beads move, bead $b^{\prime}$ is moved to the position vacated by bead $b$ and a bead is moved into the position vacated by bead $b^{\prime}$.

(i) If $u$ and $u^{\prime}-u$ are both even then $w(\gamma)=t t^{\prime}$ and a partition in $\mathcal{E}(\gamma)$ can be obtained by moving each of the $t^{\prime}$ beads on runner $u^{\prime}$ down $t$ steps.

(ii) If $u$ is odd and $u^{\prime}=p-1$ then $w(\gamma)=t\left(t^{\prime}+1\right)$ and a partition in $\mathcal{E}(\gamma)$ can be obtained by moving each of the $t$ beads on runner $u$ down $t^{\prime}+1$ steps.

Proof. We prove case (i) and then indicate the changes needed for (ii). The $p$-core $\gamma$ is represented by the abacus $A$ shown below in which the first space is in position 0 and $p$ initial beads occupy positions $-p, \ldots,-1$. (If $t>t^{\prime}$ then runners $u$ and $u^{\prime}$ should be swapped.) Let $b$ and $b^{\prime}$ be the beads in positions $u$ and $u^{\prime}$ respectively and let $c$ and $c^{\prime}$ be the lowest beads on runners $u$ and $u^{\prime}$ respectively. 


\begin{tabular}{ccccccccc}
0 & $\cdots$ & $u$ & $u+1$ & $\cdots$ & $u^{\prime}-1$ & $u^{\prime}$ & $\cdots$ & $p-1$ \\
\hline$\bullet$ & $\cdots$ & $\bullet$ & $\bullet$ & $\cdots$ & $\bullet$ & $\bullet$ & $\cdots$ & $\bullet$ \\
$\circ$ & $\cdots$ & $b \bullet$ & $\circ$ & $\cdots$ & $\circ$ & $\bullet b^{\prime}$ & $\cdots$ & $\circ$ \\
$\vdots$ & & $\vdots$ & $\vdots$ & & $\vdots$ & $\vdots$ & & $\vdots$ \\
$\circ$ & $\cdots$ & $c \bullet$ & $\circ$ & $\cdots$ & $\circ$ & $\bullet$ & $\cdots$ & $\circ$ \\
$\vdots$ & & $\vdots$ & $\vdots$ & & $\vdots$ & $\vdots$ & & $\vdots$ \\
$\circ$ & $\cdots$ & $\circ$ & $\circ$ & $\cdots$ & $\circ$ & $\bullet c^{\prime}$ & $\cdots$ & $\circ$
\end{tabular}

It is clear that $t$ single-step moves of each of the $t^{\prime}$ beads between $b^{\prime}$ and $c^{\prime}$ (inclusive, starting by moving bead $c^{\prime}$ ) give an abacus representing an even partition. It remains to show that $t t^{\prime}$ moves are necessary.

Let $B$ be an abacus representing a partition in $\mathcal{E}(\gamma)$ obtained from $A$ by a fixed sequence of single-step moves. No bead in a negative position other than -1 is moved, by Proposition 3.3. If the bead labelled $d$ in position -1 is moved, then to avoid an odd gap, the beads $b$ and $b^{\prime}$ must also be moved. Raising $d$ back to position -1 and raising all the beads in positive positions in $B$ up one row now gives an abacus representing an even partition of smaller $p$-weight. Hence the odd gaps between the beads on runners $u$ and $u^{\prime}$ are removed by moving beads only on these runners.

Either bead $c$ or bead $c^{\prime}$ is the lowest bead in $B$. If $c$ is the lowest then $c$ must have been moved down at least $t^{\prime}$ steps from its position in $A$, for otherwise there are $t+t^{\prime}$ beads in at most $t+t^{\prime}-1$ rows of $B$, and so some row is of the form

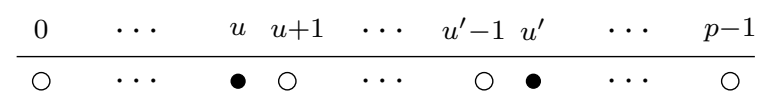

in which the beads on runners $u$ and $u^{\prime}$ form an odd gap, a contradiction. By induction $(t-1) t^{\prime}$ moves are necessary to remove all odd gaps from the abacus obtained from $A$ by deleting $c$. Hence at least $(t-1) t^{\prime}+t^{\prime}=t t^{\prime}$ moves were made to reach $B$ from $A$. The proof is similar if $c^{\prime}$ is the lowest bead in $B$.

In case (ii) a similar argument applies. Since the bead in position -1 is on runner $p-1$, it follows immediately from Proposition 3.3 that only beads on runner $u$ and runner $p-1$ are moved.

\section{Even partitions AND BlOCKS of $p$-Weight 2}

Let $\gamma$ be a $p$-core and let $\nu \in B(\gamma, 2)$. Thus $\gamma$ is obtained from $\nu$ by removing two rim $p$-hooks. In [17, page 397], Richards defines $\delta(\nu)$ to be the absolute value of the difference of the leg-lengths of these rim $p$-hooks. (This difference is independent of the choice of rim $p$-hooks.) A partition $\nu$ such that $\delta(\nu)=0$ either has two rim $p$-hooks, or a unique rim $p$-hook and 
a rim $2 p$-hook. In the former case $\nu$ is black if the larger leg-length is even, and white if it is odd. In the latter case $\nu$ is black if the leg-length of the rim $2 p$-hook is congruent to 0 or 3 modulo 4 , and otherwise white. If $\nu$ is $p$ regular, let $\nu^{\circ}$ denote the largest partition such that $\nu^{\circ} \unlhd \nu$ and $\delta\left(\nu^{\circ}\right)=\delta(\nu)$, and if $\delta(\nu)=0$, such that $\nu$ and $\nu^{\circ}$ have the same colour. (Thus $\nu^{\circ}=\nu^{\diamond^{\prime}}$ in Richards' notation.)

The main result of [17] can be stated as follows.

Theorem 4.1 (Theorem 4.4 in [17]). Let $\gamma$ be a p-core and let $\nu \in B(\gamma, 2)$ be p-regular. Then $d_{\nu \nu}=d_{\nu^{\circ} \nu}=1$. If $\mu \in B(\gamma, 2)$ and $\mu \notin\left\{\nu, \nu^{\circ}\right\}$ then $d_{\mu \nu}=1$ if $\nu^{\circ} \triangleleft \mu \triangleleft \nu$ and $\delta(\nu)-\delta(\mu) \in\{1,-1\}$; otherwise $d_{\mu \nu}=0$.

In the proof of Theorem 1.2(iii) we also need part (ii) of the following lemma. In the proof we use that the leg-length of a rim $p$-hook corresponding to a single-step upward move of a bead $b$ in position $\beta$ on an abacus is the number of beads in positions $\beta-(p-1), \ldots, \beta-1$.

Lemma 4.2. Let $\gamma$ be an even $p$-core represented by an abacus $A$ and let $\nu \in B(\gamma, 2)$.

(i) If $\nu$ is even then $\nu$ is obtained either by single-step moves of two adjacent beads in A, or by two single-step moves of the final bead in $A$.

(ii) If $\nu$ is even then $\delta(\nu)=0$.

Proof. Suppose bead $b$ on $A$ is one of the beads moved to obtain an abacus representing $\nu$. If there is a bead, say $c$, after $b$, then a new odd gap is created between the bead before $b$ in the abacus and bead $c$. Hence one of these beads must be moved. This proves (i). If the final bead is moved twice then the leg-length of the corresponding rim $2 p$-hook is 0 , so $\delta(\nu)=0$. Otherwise adjacent beads $b$ and $b^{\prime}$ are moved, where $b$ and $b^{\prime}$ are in positions $\beta$ and $\beta^{\prime}$ with $\beta<\beta^{\prime}$. Let $\mu$ be obtained from $\nu$ by removing the rim $p$-hook corresponding to $b^{\prime}$. By the observation before the lemma, the leg-lengths of the rim $p$-hooks in $\nu$ and $\mu$ corresponding to bead $b^{\prime}$ and $b$, respectively, are equal to the number of beads in $A$ in positions $\{\beta, \ldots, \beta+(p-1)\}$. Hence $\delta(\nu)=0$, as required for (ii).

It is possible to sharpen Lemma 4.2 (ii) so that the converse also holds. For this we need one more statistic. If $\nu \in B(\gamma, 2)$ is obtained by singlestep moves of beads $b$ and $b^{\prime}$ on an abacus representing $\gamma$, let $\Delta(\nu)$ be the number of beads strictly between $b$ and $b^{\prime}$. (This is clearly independent of the choice of abacus.) If $\nu$ has a rim $2 p$-hook then let $\Delta(\nu)=0$. The proof of Lemma 4.2 shows that if $\Delta(\nu)=0$ then $\delta(\nu)=0$.

Proposition 4.3. Let $\gamma$ be an even p-core and let $\nu \in B(\gamma, 2)$. Then $\nu$ is even if and only if $\Delta(\nu)=0$ and $\nu$ is black. 
Proof. Let $A$ be an abacus representing $\gamma$. Let $\nu$ be a partition obtained from $A$ by two single-step moves, represented by the abacus $B$. We consider two cases.

Case 1: Distinct beads. Suppose that distinct beads $b$ and $b^{\prime}$ are moved to obtain $B$. Let $b$ and $b^{\prime}$ be in positions $\beta$ and $\beta^{\prime}$ of $A$, respectively, where $\beta<\beta^{\prime}$. If $\nu$ is even then, by Lemma 4.2(i), $b$ and $b^{\prime}$ are adjacent and so $\Delta(\nu)=0$. Conversely, if $b$ and $b^{\prime}$ are adjacent then $\Delta(\nu)=0$. It remains to show that, if $b$ and $b^{\prime}$ are adjacent, then $\nu$ is even if and only if $\nu$ is black.

The rim $p$-hook in $\nu$ with the longer leg-length corresponds to bead $b^{\prime}$ in $B$. Its leg-length, $\ell$ say, is the number of beads in positions $\beta^{\prime}+1, \ldots, \beta+p$ of $B$. Let $a$ be the first bead before position $\beta$ in $A$. Let $e$ be the first bead before position $\beta+p$ in $B$ and let $a^{\prime}$ be the first bead after position $\beta^{\prime}+p$ in $B$; if there is no such bead let $a^{\prime}=b^{\prime}$.

If bead $e$ is bead $a$ then there are no beads except for $b$ and $b^{\prime}$ between $a$ and $a^{\prime}$ in $B$. In this case $\ell=1$ and so $\nu$ is white, and, because of the gap in $B$ between beads $a$ and $b, \nu$ is not even. In the remaining case bead $e$ is in a position $\varepsilon$ strictly between $\beta^{\prime}$ and $\beta+p$. By inserting initial beads if necessary we may assume that bead $e$ is on runner 0 , and so the relevant rows of $A$ are as shown in the abacus below, where arrows indicate the gaps in $A$ and $B$ affected by the moves of $b$ and $b^{\prime}$.

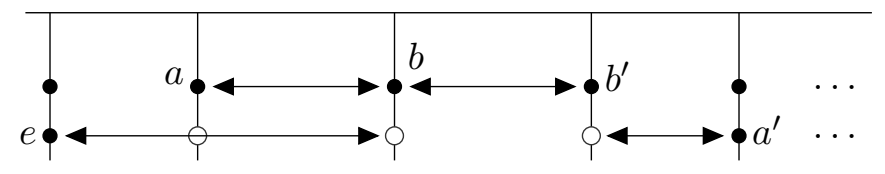

Observe that between positions $\beta$ and $\varepsilon$ (inclusive) in $A$ there are $\ell+1$ beads. Since $A$ represents an even partition, all gaps between these beads are even. Hence $\ell$ and $\varepsilon-\beta$ have the same parity and $\ell$ and $\varepsilon-\beta+p$ have opposite parity. Therefore the gap between beads $e$ and $b$ in $B$ is even if and only if $\ell$ is even, so if and only if $\nu$ is black. If there is no bead after position $\beta+p$ in $B$ then it is now clear that $\nu$ is even if and only if $\nu$ is black. Suppose that bead $a^{\prime}$ is the first bead after position $\beta+p$ in $B$. Let $a^{\prime}$ be in position $\alpha^{\prime}$. Since $A$ has a gap between positions $\varepsilon$ and $\alpha^{\prime}$, we see that

$$
\left.\alpha^{\prime}-\varepsilon=\left(\alpha^{\prime}-\left(\beta^{\prime}+p\right)\right)+\left(\beta^{\prime}-\beta\right)+((\beta+p)-\varepsilon)\right)
$$

is odd. Hence $\alpha^{\prime}-\left(\beta^{\prime}+p\right)$ and $(\beta+p)-\varepsilon$ have the same parity. Thus the gaps between beads $b$ and $e$ and beads $b^{\prime}$ and $a^{\prime}$ in $B$ have the same parity. So again we have that $\nu$ is even if and only if $\nu$ is black.

Case 2: One bead. Suppose that bead $b$ is moved twice. If $\nu$ is even then, by Lemma 4.2(i), bead $b$ is the final bead in $A$, the leg-length of the rim $2 p$-hook is 0 and $\Delta(\nu)=0$ and $\nu$ is black. 
Conversely, suppose that $\nu$ is black. Let $b$ be in position $\beta$ of $A$. Since $\delta(\nu)=0$, for each $j$ such that $1 \leq j \leq p-1$, either there are beads in both positions $\beta+j$ and $\beta+j+p$, or spaces in both these positions. Hence the leglength of the rim $2 e$-hook corresponding to bead $b$ is even, and so is congruent to 0 modulo 4 . The number of beads in positions $\beta+1, \ldots, \beta+(p-1)$ of $A$ is therefore even.

Suppose, for a contradiction, that there is a bead after position $\beta$. Let $e$ be the first bead after position $\beta$, and suppose that $e$ is in position $\varepsilon$. The positions $\beta, \ldots, \varepsilon+p$ in $A$ begin and end with beads. So we have evenly many beads in $A$ in

$$
((\varepsilon+p)-\varepsilon)+(\varepsilon-\beta)+1
$$

positions. Since $\varepsilon-\beta$ is odd, this number is odd. Therefore two of the beads in these positions form an odd gap, a contradiction. Hence $b$ is the final bead in $A$ and $\nu$ is even.

\section{Proof of Theorem 1.1}

In this section we completely characterize the vertices of all the indecomposable summands of $H^{\left(2^{n}\right)}$. The following lemma is required.

Lemma 5.1. Let $n \in \mathbf{N}$. There exist $k, \ell \in \mathbf{N}_{0}$ such that $\ell \leq(k+1)(p-1) / 2$ and

$$
2 n=(k+1)\left(2+\frac{p-1}{2} k\right)+2 \ell .
$$

Proof. For $k \in \mathbf{N}_{0}$ define $\theta_{k}=(k+1)\left(2+\frac{p-1}{2} k\right)$. Note that $\theta_{k}$ is even. Choose $k$ so that $\theta_{k} \leq 2 n<\theta_{k+1}$ and then define $\ell$ so that $(\star)$ holds. Since $\theta_{k+1}-\theta_{k}=2+(k+1)(p-1)$, we have $2 \ell \leq(k+1)(p-1)$, as required.

Proof of Theorem 1.1. By Proposition 2.1 it is sufficient to prove that every Foulkes module has a projective summand. In turn, by Proposition 2.2(i), it is sufficient to prove that for all $n$ there is an even partition $2 \lambda$ of $2 n$ with $p$-core $\gamma$ such that $2 n=|\gamma|+w(\gamma) p$, or, equivalently, such that $2 \lambda \in \mathcal{E}(\gamma)$.

We now construct such partitions. Let $k$ and $\ell$ be as in Lemma 5.1. Define

$$
\lambda_{k, 0}=(2+k(p-1), 2+(k-1)(p-1), \ldots, 2+(p-1), 2) .
$$

Note that $\lambda_{k, 0}$ is an even $p$-core partition of $(k+1)(2+(p-1) k / 2)$, represented by an abacus having $k+1$ beads on runner 2 and no beads on any other runner. Define

$$
\lambda_{k, \ell}=\lambda_{k, 0}+\left((2(s+1))^{r},(2 s)^{k+1-r}\right)
$$

where $s$ and $r$ are the unique natural numbers such that $0 \leq r<k+1$, $0 \leq s \leq \frac{p-1}{2}$ and $\ell=(k+1) s+r$. Note that $\lambda_{k, \ell}$ is an even partition of $2 n$. Let $\gamma$ be the $p$-core of $\lambda_{k, \ell}$. We consider three cases. 
(1) If $r=0$ and $0 \leq s<\frac{p-1}{2}$ then $\lambda_{k, \ell}$ is a $p$-core partition represented by an abacus with $k+1$ beads on runner $2 s+2$. Therefore $\lambda_{k, \ell}=$ $\gamma \in \mathcal{E}(\gamma)$

(2) If $r=0$ and $s=\frac{p-1}{2}$ then $\lambda_{k, \ell}$ is represented by an abacus with $k+1$ beads on runner 1 , in rows $1, \ldots, k+1$, and no beads on any other runner. Now $\lambda_{k, \ell}$ is obtained from its $p$-core $\gamma$ by the sequence of bead moves specified in Proposition 3.4(ii), taking $t=k+1, t^{\prime}=0$ and $u=1$. Therefore $\lambda_{k, \ell} \in \mathcal{E}(\gamma)$.

(3) If $r>0$ then $(k+1) s+r=\ell \leq(k+1)(p-1) / 2$ implies that $s<\frac{p-1}{2}$.

(a) If $2 s+2<p-1$ then $\lambda_{k, \ell}$ is represented by an abacus with $k+1-r$ beads on runner $2 s+2$ in rows $0, \ldots, k-r$ and $r$ beads on runner $2 s+4$ in rows $k+1-r, \ldots, k$, Now $\lambda_{k, \ell}$ is obtained from its $p$-core $\gamma$ by the sequence of bead moves specified in Proposition 3.4(i), taking $t=k+1-r, t^{\prime}=r$, $u=2 s+2$ and $u^{\prime}=2 s+4$. Therefore $\lambda_{k, \ell} \in \mathcal{E}(\gamma)$.

(b) If $2 s+2=p-1$ then $\lambda_{k, \ell}$ is represented by an abacus with $k+1-r$ beads on runner $p-1$ in rows $0, \ldots, k-r$ and $r$ beads on runner 1 in rows $k+2-r, \ldots, k+1$. Now $\lambda_{k, \ell}$ is obtained from its $p$-core $\gamma$ by the sequence of bead moves specified in Proposition 3.4(ii), taking $t=r, t^{\prime}=k+1-r, u=p-1$ and $u^{\prime}=1$. Therefore $\lambda_{k, \ell} \in \mathcal{E}(\gamma)$.

This completes the proof of Theorem 1.1 .

Remark 5.2. Let $a, n \in \mathbf{N}$. Generalizing the Foulkes modules $H^{\left(2^{n}\right)}$ already defined, let $H^{\left(a^{n}\right)}$ denote the $F S_{a n}$-module induced from the trivial representation of $S_{a} \prec S_{n}$. For $t \in \mathbf{N}_{0}$ let $P_{t}$ be a Sylow $p$-subgroup of $S_{a} \prec S_{t p}$. In [6] it is shown that if $a<p$ and $U$ is an indecomposable summand of $H^{\left(a^{n}\right)}$, then there exists $t \in \mathbf{N}_{0}$ such that $t \leq n / p$ and $P_{t}$ is a vertex of $U$. It would be interesting to know if an analogue of Theorem 1.1 holds in this general setting. More precisely, is it true that for every $t \in \mathbf{N}_{0}$ such that $t \leq n / p$, there is an indecomposable summand of $H^{\left(a^{n}\right)}$ with vertex $P_{t}$ ? The main obstacle in proving this for arbitrary $a>2$ is the lack of knowledge of the ordinary character of the Foulkes module $H^{\left(a^{n}\right)}$.

\section{Proof of Theorems 1.2 And 1.4}

Throughout this section let $\gamma$ be a $p$-core. By reduction modulo $p$, the composition factors of $H^{\left(2^{n}\right)}$ are precisely the composition factors of the Specht modules $S^{2 \lambda}$ for $\lambda$ a partition of $n$. A composition factor of $S^{2 \lambda}$ lies in the block $B(\gamma, w)$ if and only if $2 \lambda \in B(\gamma, w)$.

Blocks of $p$-weight 0 . The unique module in the block $B(\gamma, 0)$ is the simple projective Specht module $S^{\gamma}$. It is a composition factor of $H^{\left(2^{n}\right)}$ if and only 
if $\gamma$ is an even $p$-core. In this case, since $S^{\gamma}$ is projective, it splits off as a direct summand. This proves Theorem $1.2(\mathrm{i})$.

Blocks of $p$-weight 1 . Suppose that there is a summand in the block $B(\gamma, 1)$. Then an even partition can be obtained from $\gamma$ by adding a single $p$-hook. Fix an abacus representing $\gamma$. Suppose the highest odd gap is between beads $b$ and $b^{\prime}$ in positions $\beta$ and $\beta^{\prime}$ where $\beta<\beta^{\prime}$. The only singlestep moves that can lead to an even partition are moves of $b$ and $b^{\prime}$. If $b^{\prime}$ has a space below it then moving $b^{\prime}$ gives an even partition only if $b$ also has a space below it. A similar argument applies if $b$ has a space below it. Therefore both $b$ and $b^{\prime}$ have spaces below them and a single-step move of either bead gives an even partition. The two possible configurations are as shown in Figure 4 below.
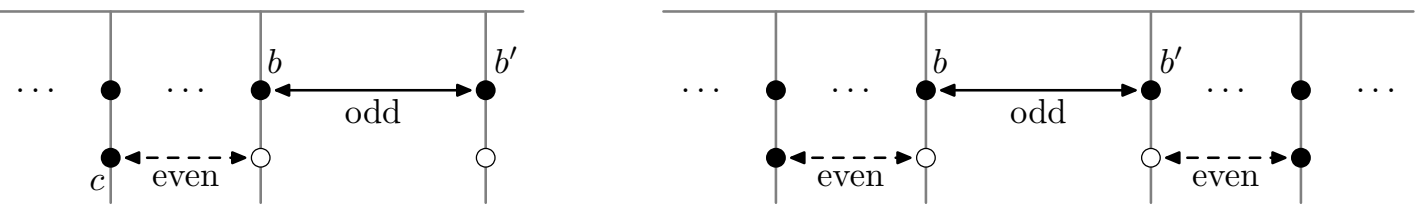

FiguRE 4. A $p$-core $\gamma$ such that $w(\gamma)=1$ has an abacus of one of the forms shown above. In the left diagram, there is a single odd gap, bead $b^{\prime}$ is on runner $p-1$, and the bead marked $c$ is the final bead on the abacus. (It is possible that $c=b^{\prime}$.) In the right diagram there are two odd gaps. In each diagram a single-step move of either $b$ or $b^{\prime}$ gives an even partition.

Let $2 \lambda$ and $2 \lambda^{\prime}$ be the even partitions obtained by moving $b$ and $b^{\prime}$, respectively. Then $\mathcal{E}(\gamma)=\left\{2 \lambda, 2 \lambda^{\prime}\right\}$. It now follows from Propositions 2.2(ii) and 2.3 that $P^{2 \lambda^{\prime}}$ is the unique summand of $H^{\left(2^{n}\right)}$ in $B(\gamma, 1)$. This completes the proof of Theorem 1.2 (iii).

Remark 6.1. By [18, $\S 6$, Example 1], $B(\gamma, 1)$ is Morita equivalent to the principal block of $F S_{p}$ by a Scopes functor. Since there are no beads between $b$ and $b^{\prime}$, the partitions $2 \lambda$ and $2 \lambda^{\prime}$ are neighbours in the dominance order on partitions in $B(\gamma, 1)$. Since Scopes functors preserve the dominance order on partitions, it follows that the Specht factors of $P^{2 \lambda^{\prime}}$ are $S^{2 \lambda^{\prime}}$ (at the top) and $S^{2 \lambda}$ (at the bottom). Thus $P^{2 \lambda^{\prime}}$ has the Loewy structure

$$
\begin{gathered}
D^{2 \lambda^{\prime}} \\
D^{\nu} \oplus D^{2 \lambda} \\
D^{2 \lambda^{\prime}}
\end{gathered}
$$

where $\nu$ is the smallest partition greater than $2 \lambda^{\prime}$ in the dominance order on partitions in $B(\gamma, 1)$. (If $2 \lambda^{\prime}$ is greatest then omit $D^{\nu}$.) 
Blocks of $p$-weight 2. Suppose that there is a summand in the block $B(\gamma, 2)$. Then either $w(\gamma)=2$, or $w(\gamma)=0$ and $\gamma$ is an even $p$-core.

Case $w(\gamma)=2$. Let $A$ be an abacus for $\gamma$. Suppose that the adjacent beads $b$ and $b^{\prime}$ form the highest odd gap in $A$. Since $\gamma$ is a $p$-core, we can assume (by adding initial beads to $A$ ) that $b$ and $b^{\prime}$ lie in the same row $r$. Suppose, for a contradiction, that neither bead $b$ nor $b^{\prime}$ is moved in a sequence of singlestep bead moves leading to an abacus $B$ representing a partition in $\mathcal{E}(\gamma)$. Then there exists a bead $c$, in an earlier position than bead $b$, that is moved into the gap between $b$ and $b^{\prime}$. Let $c^{\prime}$ be the left adjacent bead to $c$ and let $c^{\prime \prime}$ be the right adjacent bead to $c$. If $c$ is not in row $r-1$, then it is in row $r-2$ and is moved twice. This leaves an odd gap between beads $c^{\prime}$ and $c^{\prime \prime}$. Hence $c$ lies in row $r-1$. Let $A^{\prime}$ be the abacus obtained from $A$ by a single-step move of bead $c$. The odd gap between $c^{\prime}$ and $c^{\prime \prime}$ in $A^{\prime}$ cannot be removed by moving a bead $d$ from row $r-2$, since this creates a new odd gap between the two beads adjacent to $d$ in $A^{\prime}$. Hence either $c^{\prime}$ or $c^{\prime \prime}$ is moved. Therefore $B$ has two beads in the positions between $b$ and $b^{\prime}$, and so there is an odd gap in $B$ involving $b$ or $b^{\prime}$.

We have shown that either bead $b$ or bead $b^{\prime}$ is moved. Suppose that there is a bead $c$ immediately below bead $b^{\prime}$. Then there must be spaces in the positions immediately below bead $b$ and bead $c$. The partitions in $\mathcal{E}(\gamma)$ are obtained as follows:

(i) two single-step moves of bead $b$,

(ii) single-step moves of beads $b$ and $c$,

(iii) a single-step move of bead $c$ followed by a single-step move of bead $b^{\prime}$.

Hence $|\mathcal{E}(\gamma)|=3$ and the unique maximal partition in $\mathcal{E}(\gamma)$ is the partition $2 \lambda$ given by the moves in (iii). By Propositions 2.2(ii) and 2.3, $P^{2 \lambda}$ is the unique summand of $H^{\left(2^{n}\right)}$ in $B(\gamma, 2)$. A similar result holds if there is a bead immediately below bead $b$; in this case the unique maximal partition is given by two single-step moves of bead $b^{\prime}$. (This case occurs in the block $B((3,1), 2)$ when $p=3$ in the example in $\S 8$ below. $)$

Now suppose there are spaces below beads $b$ and $b^{\prime}$. Let $\mu$ be the partition obtained by a single-step move of bead $b^{\prime}$, represented by the abacus $B$. Let $C$ be the abacus obtained by swapping the bead $b$ on $B$ with the space in $B$ in the position occupied by bead $b^{\prime}$ in $A$. Let $\gamma^{\star}$ be the $p$-core represented by $C$. The abaci $B$ and $C$ have the same pattern of odd and even gaps. Moreover, a single-step move of bead $b$ on $B$ does not give an even partition, since it restores the odd gap between beads $b$ and $b^{\prime}$ present in $A$. Therefore $w\left(\gamma^{\star}\right)=1$ and there is a bijection between the sequences of single-step moves on $B$ and on $C$ that give even partitions. From the $p$-weight one case we have $\mathcal{E}\left(\gamma^{\star}\right)=2$. Hence exactly two even partitions can be obtained by 
starting with a single-step move of bead $b^{\prime}$, leaving bead $b$ fixed. A similar argument deals with the case where bead $b$ is moved. Therefore $|\mathcal{E}(\gamma)|=4$, and by Propositions 2.2(ii) and 2.3 , it follows that if $2 \lambda$ is a maximal element of $\mathcal{E}(\gamma)$ then $P^{2 \lambda}$ is the unique summand of $H^{\left(2^{n}\right)}$ in $B(\gamma, 2)$.

Example 6.2. We pause to give an example of the case where $w(\gamma)=2$ and there are spaces below beads $b$ and $b^{\prime}$. Let $p=5$ and let $\gamma=(5,1,1,1)$. An abacus $A$ representing $\gamma$, and the abacus $C$ defined in the proof, are shown below left and below right.

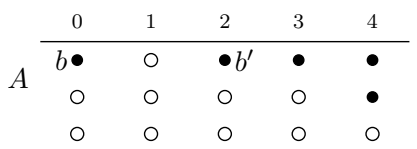

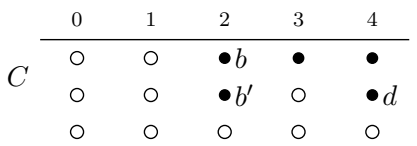

Moving bead $b^{\prime}$ or bead $d$ in $C$ gives an even partition. The corresponding elements of $\mathcal{E}(\gamma)$, obtained by moving bead $b$ in $C$ back to position 0 are $(8,6,2,2)$ and $(10,4,2,2)$, respectively. The other partitions in $\mathcal{E}(\gamma)$ are found by moving $b$ first; they are $(6,6,2,2,2)$ and $(10,2,2,2,2)$. Thus the unique summand of $H^{\left(2^{9}\right)}$ in $B(\gamma, 2)$ is $P^{(10,4,2,2)}$.

Case $w(\gamma)=0$. Since $H^{\left(2^{n-p}\right)}$ has $S^{\gamma}$ as its unique summand in the block $B(\gamma, 0)$, it follows from Proposition 2.1 that there is a unique summand of $H^{\left(2^{n}\right)}$ in $B(\gamma, 2)$ with vertex $Q_{1}$. Since any summand in $B(\gamma, 2)$ has a vertex contained in the $p$-weight 2 defect group $\langle(1,2, \ldots, p),(p+1, \ldots, 2 p)\rangle$ (see [13. Theorem 6.2.45]), any other summand in this block must be projective.

Suppose that $P^{\nu}$ is such a projective summand. By Proposition 2.2(iii) $\nu$ is an even partition. By Lemma 4.2(ii), $\delta(\nu)=0$. By Theorem 4.1 and Proposition 2.3, the column of the decomposition matrix labelled by $\nu$ has a non-zero entry in a row labelled by partition $\mu$ with $\delta(\mu)=1$. By another application of Lemma 4.2(ii), this partition $\mu$ is not even, contradicting Proposition 2.2(iii). This completes the proof.

As a corollary, we are now ready to deduce Theorem 1.4

Proof of Theorem 1.4. Let $t=\lfloor n / p\rfloor$ and let $r=n-t p$. Since $Q_{t}$ permutes $2 t p$ points, if a block $B(\gamma, w)$ of $S_{2 n}$ contains a summand of $H^{\left(2^{n}\right)}$ with vertex $Q_{t}$ then $w p \geq 2 t p$, and so $w=2 t$. Let $\gamma$ be a $p$-core such that $|\gamma|+2 t p=2 n$. By Proposition 2.1, the number of indecomposable summands of $H^{\left(2^{n}\right)}$ with vertex $Q_{t}$ in $B(\gamma, 2 t)$ is equal to the number of indecomposable projective summands of $H^{\left(2^{r}\right)}$ in the block $B(\gamma, w-2 t)$ of $S_{2 r}$. Since $r<$ $p$, it follows from Theorem 1.2 that $H^{\left(2^{r}\right)}$ has at most one summand in $B(\gamma, w-2 t)$, and that every such summand is projective. Hence the number of indecomposable summands of $H^{\left(2^{n}\right)}$ with vertex $Q_{t}$ is equal to the number of blocks of $H^{\left(2^{r}\right)}$ containing an even partition. This equals the number 
of $p$-core partitions that can be obtained by removing $p$-hooks from even partitions of $2 r$, as required.

\section{Proof of Theorem 1.3}

Let $2 n=2 k+2 p$. By hypothesis $0 \leq 2 k<p$. The $p$-core $(2 k)$ is represented by the abacus shown below. (If $k=0$ then the bead in the second row should be deleted.)

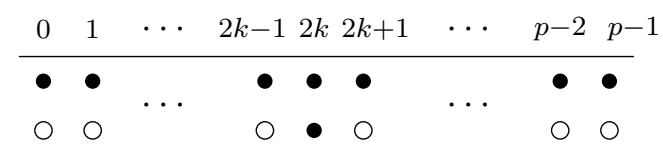

Let $\langle u\rangle$ denote the partition obtained by two single-step moves of the lowest bead on runner $u$. Let $\langle u, u\rangle$ denote the partition obtained by a single-step move of the lowest bead on runner $u$ followed by a single-step move of the bead immediately above it. Finally let $\langle u, v\rangle$ denote the partition obtained by single-step moves of the lowest beads on runners $u$ and $v$.

It follows from Proposition 4.3 , but can also easily be seen directly, that if $k \neq 0$ then the even partitions in $B((2 k), 2)$ are $\langle 2 k\rangle,\langle p-1\rangle$ and $\langle j, j+1\rangle$ where either $j<2 k$ and $j$ is even, or $j>2 k$ and $j$ is odd. If $k=0$ then they are $\langle p-1\rangle$ and $\langle j, j+1\rangle$ for $j$ even. A convenient way to find the composition factors of the corresponding Specht modules uses the chains in the following lemma.

Lemma 7.1. Let $\mathcal{P}$ be the set consisting of all even partitions in $B((2 k), 2)$ together with all partitions $\nu \in B((2 k), 2)$ such that $\delta(\nu)=1$. Then $\mathcal{P}$ is totally ordered by the dominance order. The elements of $\mathcal{P}$ are as follows. If $2<2 k<p-1$ then

$$
\begin{aligned}
& \langle\mathbf{2} \mathbf{k}\rangle \triangleright\langle p-1\rangle \triangleright\langle 2 k, p-2\rangle \triangleright\langle\mathbf{p}-\mathbf{2}, \mathbf{p}-\mathbf{1}\rangle \triangleright \cdots \\
& \cdots \triangleright\langle\mathbf{2} \mathbf{t}+\mathbf{1}, \mathbf{2} \mathbf{t}+\mathbf{2}\rangle \triangleright\langle 2 t, 2 t+2\rangle \triangleright\langle 2 t-1,2 t+1\rangle \triangleright\langle\mathbf{2} \mathbf{t}-\mathbf{1}, \mathbf{2} \mathbf{t}\rangle \triangleright \cdots \\
& \cdots \triangleright\langle\mathbf{2} \mathbf{k}+\mathbf{1}, \mathbf{2} \mathbf{k}+\mathbf{2}\rangle \triangleright\langle 2 k-1,2 k+2\rangle \triangleright\langle 2 k-2,2 k+1\rangle \triangleright\langle\mathbf{2} \mathbf{k}-\mathbf{2}, \mathbf{2 k}-\mathbf{1}\rangle \triangleright \cdots \\
& \cdots \triangleright\langle\mathbf{2} \mathbf{s}, \mathbf{2}+\mathbf{1}\rangle \triangleright\langle 2 s-1,2 s+1\rangle \triangleright\langle 2 s-2,2 s\rangle \triangleright\langle\mathbf{2} \mathbf{s}-\mathbf{2}, \mathbf{2} \mathbf{s}-\mathbf{1}\rangle \triangleright \cdots \\
& \cdots \triangleright\langle\mathbf{2}, \mathbf{3}\rangle \triangleright\langle 1,3\rangle \triangleright\langle 0,2\rangle \triangleright\langle\mathbf{0}, \mathbf{1}\rangle \triangleright\langle 1,1\rangle ;
\end{aligned}
$$

if $2 k=0$ then

$$
\begin{array}{r}
\langle\mathbf{p}-\mathbf{1}\rangle \triangleright\langle p-2\rangle \triangleright\langle p-3, p-1\rangle \triangleright\langle\mathbf{p}-\mathbf{3}, \mathbf{p}-\mathbf{2}\rangle \triangleright\langle p-4, p-2\rangle \triangleright \cdots \\
\cdots \triangleright\langle 2,4\rangle \triangleright\langle\mathbf{2}, \mathbf{3}\rangle \triangleright\langle 1,3\rangle \triangleright\langle 0,2\rangle \triangleright\langle\mathbf{0}, \mathbf{1}\rangle \triangleright\langle 1,1\rangle
\end{array}
$$


with the general case as above; if $2 k=2$ then

$$
\begin{aligned}
\langle\mathbf{2}\rangle \triangleright\langle p-1\rangle \triangleright\langle 2, p-2\rangle & \triangleright\langle\mathbf{p}-\mathbf{2}, \mathbf{p}-\mathbf{1}\rangle \triangleright\langle p-3, p-1\rangle \triangleright \cdots \\
\cdots & \triangleright\langle 3,5\rangle \triangleright\langle\mathbf{3}, \mathbf{4}\rangle \triangleright\langle 1,4\rangle \triangleright\langle 0,3\rangle \triangleright\langle\mathbf{0}, \mathbf{1}\rangle \triangleright\langle 1,1\rangle
\end{aligned}
$$

with the general case as above; and if $2 k=p-1$ then

$$
\begin{aligned}
\langle\mathbf{p}-\mathbf{1}\rangle \triangleright\langle p-2, p-1\rangle & \triangleright\langle p-3\rangle \triangleright\langle\mathbf{p}-\mathbf{3}, \mathbf{p}-\mathbf{2}\rangle \triangleright\langle p-4, p-2\rangle \triangleright \cdots \\
& \cdots \triangleright\langle 2,4\rangle \triangleright\langle\mathbf{2}, \mathbf{3}\rangle \triangleright\langle 1,3\rangle \triangleright\langle 0,2\rangle \triangleright\langle\mathbf{0}, \mathbf{1}\rangle \triangleright\langle 1,1\rangle
\end{aligned}
$$

with the general case as above. The even partitions are shown in bold type.

Proof. It is routine to check that the partitions $\nu$ such that $\delta(\nu)=1$ are as claimed. Lemma 4.4 in [17, which states that $\langle u, v\rangle \unlhd\left\langle u^{\prime}, v^{\prime}\right\rangle$ if and only if $u \leq u^{\prime}$ and $v \leq v^{\prime}$, then gives the total order of the chains, except for the cases involving partitions of the form $\langle u\rangle$, which have to be checked separately.

Proposition 7.2. Let $\mu \in B((2 k), 2)$ be even. Suppose that, in the relevant chain of partitions in Lemma 7.1, we have adjacent partitions $\mu^{\prime} \triangleright \nu^{\prime} \triangleright \nu \triangleright$ $\mu \triangleright \nu^{\prime \prime}$ where $\mu^{\prime}$ is even. Then

(i) $\mu$ is even and $\mu^{\prime \circ}=\mu$ and $\nu^{\circ}=\nu^{\prime \prime}$;

(ii) if $\mu \neq\langle 0,1\rangle$ then the composition factors of $S^{\mu}$ are $D^{\mu^{\prime}}, D^{\nu}$ and $D^{\mu}$;

(iii) the composition factors of $S^{\langle 0,1\rangle}$ are $D^{\langle 2,3\rangle}$ and $D^{\langle 0,2\rangle}$;

(iv) $\operatorname{Ext}^{1}\left(D^{\mu^{\prime}}, D^{\nu}\right)=\operatorname{Ext}^{1}\left(D^{\nu}, D^{\mu}\right)=F$;

(v) if $\lambda \in B((2 k), 2)$ and $\operatorname{Ext}^{1}\left(D^{\mu}, D^{\lambda}\right) \neq 0$ then $\delta(\lambda)=1$.

Proof. Part (i) follows from the definition of the map $\circ$ and inspection of the chains in Lemma 7.1. Then (ii) and (iii) are easy deductions from Lemma 7.1 and Theorem 4.1. The Ext quivers of $p$-weight two principal blocks of symmetric groups were found by Martin in [15]: parts (iv) and (v) can be read off from Figures 9 and 10 in the appendix.

We are now ready to prove Theorem 1.3 .

Proof of Theorem 1.3. By Theorem 1.2(iii), there is a unique indecomposable summand of $H^{\left(2^{p+k}\right)}$ in the block $B((2 k), 2)$ of $S_{2(p+k)}$. This summand, $U$ say, is the Scott module of vertex $Q_{1}$. By [3, (2.1)], $U$ is self-dual and has the trivial module in its socle. By Proposition 7.2(ii) and (iii), each $D^{\nu}$ labelled by a partition $\nu$ such that $\delta(\nu)=1$ appears exactly once in $U$. Hence if $2<2 k<p-1$ then the heart of $U$ is

$$
\begin{aligned}
D^{\langle 2 k, p-2\rangle} & \oplus D^{\langle p-4, p-2\rangle} \oplus \cdots \\
& \cdots \oplus D^{\langle 2 k+1,2 k+3\rangle} \oplus D^{\langle 2 k-2,2 k+1\rangle} \oplus D^{\langle 2 k-4,2 k-2\rangle} \oplus \cdots \oplus D^{\langle 0,2\rangle} .
\end{aligned}
$$


If $2 k \in\{0,2, p-1\}$ then an analogous result holds with minor changes to the labels, as indicated in Figure 5 below. Similarly, each simple module $D^{2 \lambda}$ labelled by a $p$-regular even partition $2 \lambda$ appears exactly twice in $U$. By Proposition 7.2(iv), the only extensions in $U$ are between modules in the heart of $U$ and these $D^{2 \lambda}$. It is easily seen, either from the chains in Lemma 7.1, or from Lemma 4.3 in [17] and our Lemma 4.2, that the unique even partition in $B((2 k), 2)$ that is not $p$-regular is $\left(2 k, 2^{p}\right)$ (interpreted as $\left(2^{p}\right)$ if $\left.k=0\right)$. Hence $U$ has three Loewy layers and

$$
\operatorname{soc} U \cong \operatorname{top} U \cong \bigoplus_{\substack{2 \lambda \in B((2 k), 2) \\ 2 \lambda \neq\left(2 k, 2^{P}\right)}} D^{2 \nu} .
$$

If $2<2 k<p-1$ then the structure of $U$ is as shown in Figure 1. The exceptional cases are shown in Figure 5 below.

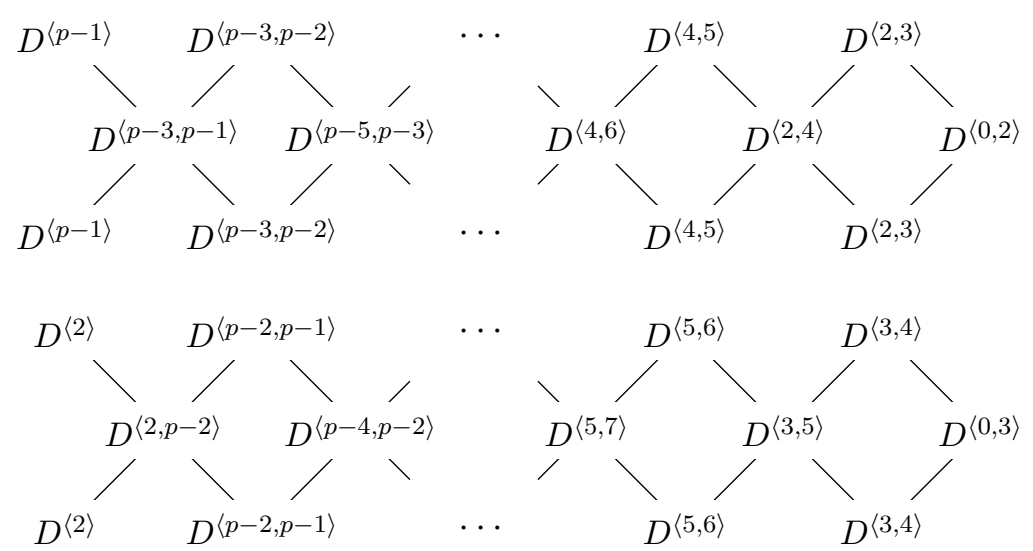

FiguRE 5. Loewy layers of the Scott module summand $U$ of $H^{(2 n)}$ in the block $B((2 k), 2)$ in the cases $2 k=0$ (top) and $2 k=2$ (bottom). If $2 k=p-1$ then the diagram for $2 k=0$ applies, replacing $\langle p-3, p-1\rangle$ with $\langle p-3\rangle$.

Remark 7.3. In [16], Paget proved that $H^{\left(2^{n}\right)}$ has a filtration $0=V_{0} \subset$ $V_{1} \subset \ldots \subset V_{d}=H^{\left(2^{n}\right)}$ such that $V_{i} / V_{i-1} \cong S^{2 \lambda(i)}$, where $\lambda(1)<\ldots<\lambda(d)$ are the partitions of $n$. By Proposition 13 in [19], which was proved using the Hemmer-Nakano homological characterization of modules with a Specht filtration (see [9]), it follows that the summand $U$ has a Specht filtration, provided $p \geq 5$. This filtration can be seen in Figures 1 and 5 : for $2 k>2$, the bottom Specht factor is $S^{\langle 0,1\rangle}$ (with composition factors $D^{\langle 0,2\rangle}$ and $D^{\langle 2,3\rangle}$ ) the next is $S^{\langle 2,3\rangle}$ (with composition factors $D^{\langle 2,3\rangle}, D^{\langle 2,4\rangle}, D^{\langle 4,5\rangle}$ ), and on on, ending with the trivial module $D^{\langle 2 k\rangle}$ at the top. 
It would be interesting to know other cases where a single indecomposable module exhibits a significant proportion of the extensions between two classes of simple modules for the symmetric group.

\section{EXAmple: $H^{\left(2^{7}\right)}$ IN CharaCteristic 5}

Let $p=5$. The even partitions of 14 lie in the 5-blocks $B((4), 2)$, $B((2,2), 2), B((3,1), 2), B\left(\left(1^{4}\right), 2\right), B((5,2,2), 1)$ and $B((4,4,2,2,2), 0)$ of $S_{14}$. The principal block summand, $U$ say, is dealt with by Theorem 1.3 . Since $w((3,1))=w\left(\left(1^{4}\right)\right)=2, w((5,2,2))=1$ and $w((4,4,2,2,2))=0$, it follows from Theorem 1.2 that the summands in these blocks are the projective modules $P^{(10,4)}, P^{(8,2,2,2)}, P^{(10,2,2)}$ and $S^{(4,4,2,2,2)}$, respectively. By Theorem 1.2(iii), there is a unique summand, $V$ say, in $B((2,2), 2)$. This summand has vertex $Q_{1}$. By Remark 7.3 , $V$ has a Specht filtration by the Specht factors in this block, namely $S^{(12,2)}, S^{(6,4,4)}$ and $S^{\left(2^{7}\right)}$. Using Theorem 4.1 to get the required decomposition numbers, one finds that

$$
\begin{aligned}
& U \oplus V \oplus P^{(10,4)} \oplus P^{(8,2,2,2)} \\
&=\left(\begin{array}{c}
S^{(14)} \\
S^{(4,4,4,2)} \\
S^{\left(4,2^{5}\right)}
\end{array}\right) \bigoplus\left(\begin{array}{c}
S^{(12,2)} \\
S^{(6,4,4)} \\
S^{\left(2^{7}\right)}
\end{array}\right) \bigoplus\left(\begin{array}{c}
S^{(10,4)} \\
S^{(8,6)} \\
S^{(8,4,2)}
\end{array}\right) \bigoplus\left(\begin{array}{c}
S^{(8,2,2,2)} \\
S^{(6,4,2,2)} \\
S^{\left(6,2^{4}\right)}
\end{array}\right) \\
&=\left(\begin{array}{c}
D^{(14)} \oplus D^{(4,4,4,2)} \\
D^{(7,5,1,1)} \oplus D^{(4,3,2,2,2,1)} \\
D^{(14)} \oplus D^{(4,4,4,2)}
\end{array}\right) \bigoplus\left(\begin{array}{c}
D^{(12,2)} \oplus D^{(6,4,4)} \\
D^{(7,4,3)} \oplus D^{(3,3,3,2,1,1,1)} \\
D^{(12,2)} \oplus D^{(6,4,4)}
\end{array}\right) \\
& \bigoplus\left(\begin{array}{c}
D^{(10,4)} \\
D^{(13,1)} \oplus D^{(8,6)} \\
D^{(10,4)} \oplus D^{(8,4,2)} \\
D^{(13,1)} \oplus D^{(8,6)} \\
D^{(10,4)}
\end{array}\right) \bigoplus\left(\begin{array}{c}
D^{(8,2,2,2)} \\
D^{(9,2,2,1)} \oplus D^{(6,4,2,2)} \\
D^{(6,5,2,1)} \oplus D^{(8,2,2,2)} \oplus D^{(6,2,2,2,2)} \\
D^{(9,2,2,1)} \oplus D^{(6,4,2,2)} \\
D^{(8,2,2,2)}
\end{array}\right) .
\end{aligned}
$$

The Loewy layers of $P^{(10,2,2)}$ are given by Remark 6.1 , while the other summand, $S^{(4,4,2,2,2)}$ is simple. By Proposition 6.5 in [7], all these summands have abelian endomorphism rings. We note that $P^{(10,4)}$ is the projective summand used in the proof of Theorem 1.1.

\section{REFERENCES}

[1] J. L. Alperin, Local representation theory, Cambridge studies in advanced mathematics, vol. 11, CUP, 1986.

[2] C. Bessenrodt and K. Uno, Character relations and simple modules in the AuslanderReiten graph of the symmetric and alternating groups and their covering groups, Algebr. Represent. Theory 4 (2001), no. 5, 445-468.

[3] M. Broué, On Scott modules and p-permutation modules: an approach through the Brauer homomorphism, Proc. Amer. Math. Soc. 93 (1985), 401-408. 
[4] C. Gill, Young module multiplicities, decomposition numbers and the indecomposable Young permutation modules, J. Algebra Appl., 13 (2014), 1350147, 23 pp.

[5] K. Erdmann, Young modules for symmetric groups, J. Aust. Math. Soc. 71 (2001), 201-210.

[6] E. Giannelli, On permutation modules and decomposition numbers for symmetric groups, J. Algebra 312 (2015) pp. 427-449.

[7] E. Giannelli and M. Wildon, Foulkes modules and decomposition numbers of the symmetric group, J. Pure Appl. Algebra 219 (2015), no. 2, 255-276.

[8] J. A. Green, Multiplicities, Scott modules and lower defect groups, J. London Math. Soc. (2) 28 (1983), no. 2, 282-292.

[9] D. J. Hemmer and D. K. Nakano, Specht filtrations for Hecke algebras of type A, J. London Math. Soc. (2) 69 (2004), 623-638.

[10] A. Henke, On p-Kostka numbers and Young modules, European J. Combin. 26 (2005), 923-942.

[11] N. F. Inglis, R. W. Richardson, and J. Saxl, An explicit model for the complex representations of $S_{n}$, Arch. Math. (Basel) 54 (1990), 258-259.

[12] G. D. James, Trivial source modules for symmetric groups, Arch. Math. (Basel), 41 (1983), 294-300.

[13] G. James and A. Kerber, The representation theory of the symmetric group, Encyclopedia of Mathematics and its Applications, vol. 16, Addison-Wesley Publishing Co., Reading, Mass., 1981.

[14] G. D. James, The representation theory of the symmetric groups, Lecture Notes in Mathematics, vol. 682, Springer, Berlin, 1978.

[15] S. Martin, D. Phil thesis, Oxford, 1988.

[16] R. Paget, A family of modules with Specht and dual Specht filtrations, J. Algebra 312 (2007), no. 2, 880-890.

[17] M. J. Richards, Some decomposition numbers for Hecke algebras of general linear groups, Math. Proc. Cambridge Philos. Soc. 119 (1996), no. 3, 383-402.

[18] J. Scopes, Cartan matrices and Morita equivalence for blocks of the symmetric groups, J. Algebra 142 (1991), 441-455.

[19] M. Wildon, Multiplicity-free representations of symmetric groups, J. Pure Appl. Alg. 213 (2009), 1464-1477.

(E. Giannelli) Department of Mathematics, University of Kaiserslautern, P.O. Box 3049, 67655 Kaiserslautern, Germany

E-mail address: gianelli@mathematik.uni-kl.de

(M. Wildon) Department of Mathematics, Royal Holloway, University of LONDON, United Kingdom

E-mail address: mark.wildon@rhul.ac.uk 